\title{
DỨBin
}

Technological University Dublin

ARROW@TU Dublin

Photonics Research Centre

2019

\section{Mode Transition in Conventional Step-Index Optical Fibers}

\author{
Xiaokang Lian \\ Technological University Dublin \\ Gerald Farrell \\ Technological University Dublin, gerald.farrell@tudublin.ie \\ Qiang Wu \\ Northumbria University
}

See next page for additional authors

Follow this and additional works at: https://arrow.tudublin.ie/prccon

Part of the Electrical and Computer Engineering Commons, Engineering Physics Commons, and the Optics Commons

\section{Recommended Citation}

X. Lian, G. Farrell, Q. Wu, W. Han, F. Wei and Y. Semenova, "Mode Transition in Conventional Step-Index Optical Fibers," 2019 18th International Conference on Optical Communications and Networks (ICOCN), 2019, pp. 1-3, doi: 10.1109/ICOCN.2019.8934113.

This Conference Paper is brought to you for free and open access by the Photonics Research Centre at ARROW@TU Dublin. It has been accepted for inclusion in Conference Papers by an authorized administrator of ARROW@TU Dublin. For more information, please contact arrow.admin@tudublin.ie, aisling.coyne@tudublin.ie,gerard.connolly@tudublin.ie. Funder: Technological University Dublin

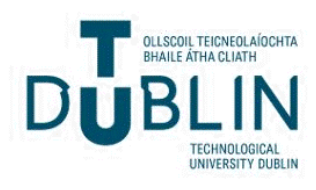


Authors

Xiaokang Lian, Gerald Farrell, Qiang Wu, Wei Han, Fangfang Wei, and Yuliya Semenova 


\title{
Mode Transition in Conventional Step-Index Optical Fibers
}

\author{
Xiaokang, Lian ${ }^{1 *}$, Gerald Farrell ${ }^{1}$, Qiang $W^{2}$, Wei Han ${ }^{1}$, Fangfang Wei ${ }^{1}$, and Yuliya Semenova ${ }^{1}$. \\ ${ }^{1}$ Photonics Research Centre, Technological University Dublin, Dublin D08 X622, Ireland. \\ ${ }^{2}$ Department of Mathematics, Physics, and Electrical Engineering, Northumbria University, Newcastle upon Tyne NE1 \\ 8ST, U.K. \\ *email: Xiaokang.lian@mydit.ie
}

\begin{abstract}
The discrete self-imaging effect reveals the distinct properties of cladding modes with core modes in step-index optical fibers, as was shown in our previous work [1], where only the linearly polarized $L P_{0 n}$ modes were studied. In this paper, the dispersion diagram of the first 17 vector modes $\left(T E_{0 n}, T M_{0 n}, H E_{m n}\right.$ and $\left.E H_{m n}\right)$ and the related first $9 L P_{m n}$ modes are calculated by both the full-vector finite element method and the graphical method with a three-layer step-index optical fiber model. The cladding modes distributions and the transitions between the core and cladding modes are analyzed. The results of this work are intended to enrich the knowledge and understanding of modal characteristics of conventional step-index optical fibers.
\end{abstract}

Keywords: Optical fiber dispersion, mode transition, optical fiber cladding, step-index optical fiber, Talbot and self-imaging effect.

\section{INTRODUCTION}

Silica-based solid core step-index optical fibers such as the single-mode optical fiber (SMF) are widely used as the fundamental component in the telecommunications industry. Their single-step index profile is the simplest fiber design and provides a logical starting point for the study of fibers with more complex designs [1]-[6]. For example, the classification of the modes in the complex hollow-core photonic-bandgap fibers is similar to that in the step-index optical fibers, although the guidance mechanism in the former is the photonic bandgap effect unlike the total internal reflection effect in the latter [7].

Most of the research relating to step-index optical fibers has focused on the studies of the core modes. The two-layer model (the single-step index profile) used in studies of the core modes assumes that the cladding region is infinite, therefore, the information related to the cladding modes cannot be obtained. The cladding modes could be studied with a three-layer model (a double-step index profile) and their modal characteristic equations have been derived by several different groups [3]-[5]. The cladding modes can be excited by fiber Bragg gratings (FBGs) and long-period fiber gratings (LPFGs), by coupling the energy of core modes into cladding modes. The theory relating to the mode coupling in the fiber gratings has been well described in literature [5], [6]. However, an intuitive interpretation of the qualitative and quantitative characteristics of the cladding modes is still lacking. In this paper, a complete dispersion diagram including the core and cladding modes is developed, which enriches the knowledge and understanding of modal characteristics in conventional step-index optical fibers.

\section{METHODS}

Fig. 1(a) shows the cross section and the index profile of a step-index optical fiber in a three-layer model. The refractive index of the fiber core $n_{\mathrm{co}}$ is higher than that of the fiber cladding $n_{\mathrm{cl}}$, where the radius of the core is $r_{\mathrm{co}}$ and the cladding radius is $r_{\mathrm{cl}}$. The surrounding is air, with the minimum refractive index $n_{\text {air }}$. The effective refractive index $n_{\text {eff }}$ of the core-type modes lies within a range defined by $n_{\mathrm{co}}>n_{\mathrm{eff}}>n_{\mathrm{cl}}$ while for the cladding modes it is $n_{\mathrm{cl}}>n_{\mathrm{eff}}>n_{\text {air. }}$. In the ray optics model as shown in Figs. 1(b) and 1(c), the ray of the core mode is confined inside the core region by the total internal reflection at the interface between the core and cladding regions. For the cladding mode, the ray can partially penetrate the core-cladding interface in the fashion of a multiple-path reflection while the total internal reflection only occurs at the interface between the cladding region and the surrounding medium (air).

In the calculations, the fiber parameters used are the same as in our previous work [1]: $n_{\mathrm{co}}=1.451, n_{\mathrm{cl}}=$ $1.445, r_{\mathrm{cl}}=62.5 \mu \mathrm{m}, n_{\mathrm{air}}=1.000$ and the light wavelength is $\lambda=1550 \mathrm{~nm}$. The core radius $r_{\mathrm{co}}$ is the only variable, covering the entire radial range from $0 \mu \mathrm{m}$ to $62.5 \mu \mathrm{m}$. As discussed in Ref. [1], the linearly polarized (LP) approximation is suitable over the entire $r_{\text {co }}$ range. The LP approximation is reasonable due to a relatively small difference between the core and cladding refractive indices, $n_{\mathrm{co}}(1.451)$ and $n_{\mathrm{cl}}(1.445)$. It is also suitable for the no-core fibers (NCFs) due to the mode fields being far from cutoff, although the difference between $n_{\mathrm{cl}}(1.445)$ and $n_{\text {air }}(1.000)$ is relatively large. The $n_{\text {eff }}$ of the scalar $\mathrm{LP}_{\mathrm{mn}}$ modes are calculated by the graphical method with the scalar eigenvalue equations [4]. The $n_{\text {eff }}$ of the vector modes $\left(\mathrm{TE}_{0 \mathrm{n}}, \mathrm{TM}_{0 \mathrm{n}}, \mathrm{HE}_{\mathrm{mn}}\right.$ and $\left.\mathrm{EH}_{\mathrm{mn}}\right)$ are calculated by the full-vector finite element method (FEM). The subscript $\mathrm{m}$ is the azimuthal number and $\mathrm{n}$ is the radial number. 
(a)

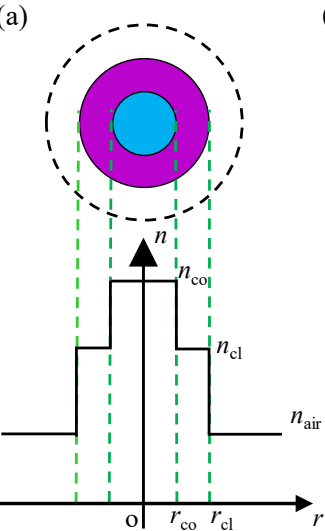

(b)

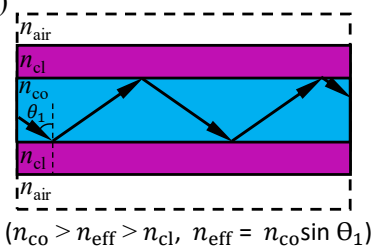

(c)

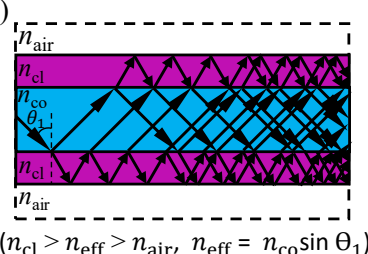

(n)

$\left(n_{\mathrm{cl}}>n_{\mathrm{eff}}>n_{\mathrm{air}}, n_{\mathrm{eff}}=n_{\mathrm{co}} \sin \theta_{1}\right)$

Fig. 1. (a) The refractive index profile of a three-layer step-index optical fiber. (b) Ray trajectory of a core-type mode. (c) Ray trajectory of a cladding-type mode. $\left(0 \leq r_{\mathrm{co}} \leq 62.5 \mu \mathrm{m}, n_{\mathrm{co}}=1.451, r_{\mathrm{cl}}=62.5 \mu \mathrm{m}\right.$ and $n_{\mathrm{cl}}=1.445, \lambda=1550 \mathrm{~nm}$ )

\section{RESULTS}

The dispersion diagrams ( $n_{\mathrm{eff}}$ vs. $r_{\mathrm{co}}$ ), including the first 17 vector modes $\left(\mathrm{TE}_{0 \mathrm{n}}, \mathrm{TM}_{0 \mathrm{n}}, \mathrm{HE}_{\mathrm{mn}}\right.$ and $\left.\mathrm{EH}_{\mathrm{mn}}\right)$ and the first 9 scalar $\mathrm{LP}_{\mathrm{mn}}$ modes, are shown in Fig. 2. The corresponding $V$-parameter values are shown as the top axis of the graphs, for which the expression is:

$$
V=\frac{2 \pi r_{\mathrm{co}}}{\lambda} \sqrt{n_{\mathrm{co}}^{2}-n_{\mathrm{cl}}^{2}}
$$

The $n_{\text {eff }}$ of the scalar $\mathrm{LP}_{\mathrm{mn}}$ modes calculated by the graphical method are shown as the real lines while those of vector modes calculated by the full-vector FEM are shown as the scattered lines. The vector modes are only calculated in a small range of core radii $0 \mu \mathrm{m} \leq r_{\mathrm{co}} \leq 15$ $\mu \mathrm{m}$ for conciseness, in order to show the relationship with the scalar $\mathrm{LP}_{\mathrm{mn}}$ modes. $\mathrm{LP}_{0 \mathrm{n}}$ is a doubly degenerate mode of two $\mathrm{HE}_{1 \mathrm{n}}$ modes; the $\mathrm{LP}_{1 \mathrm{n}}$ is a four-fold degenerate mode of $\mathrm{TE}_{0 \mathrm{n}}, \mathrm{TM}_{0 \mathrm{n}}$, and two $\mathrm{HE}_{2 \mathrm{n}}$ modes; $\mathrm{LP}_{\mathrm{mn}}(\mathrm{m}>1)$ is a four-fold degenerate mode of two $\mathrm{HE}_{\mathrm{m}+1, \mathrm{n}}$ and two $\mathrm{EH}_{\mathrm{m}-1, \mathrm{n}}$ modes. The $n_{\mathrm{eff}}$ of every vector mode in each group are almost same as those of the corresponding $\mathrm{LP}_{\mathrm{mn}}$ modes, as seen from the overlapped scatters and the real lines in Fig. 2. The excellent match between the $n_{\text {eff }}$ of the scalar modes and the vector modes verifies the LP approximation. The mode distribution of the vector modes is the same as for the corresponding $\mathrm{LP}_{\mathrm{mn}}$ modes.

The Fig. 2(a) is divided into two parts by the red dash horizontal lines located at $n_{\mathrm{co}}$ and $n_{\mathrm{cl}}$. The region with $n_{\mathrm{cl}}<n_{\mathrm{eff}}<n_{\mathrm{co}}$ corresponds to the fiber core modes while the region with $n_{\mathrm{eff}}<n_{\mathrm{cl}}\left(r_{\mathrm{co}} \neq 0 \mu \mathrm{m}\right)$ corresponds to the fiber cladding modes. All the modes in NCFs with $r_{\mathrm{co}}=0 \mu \mathrm{m}$ or $r_{\mathrm{co}}=62.5 \mu \mathrm{m}$ are classified as core modes, since there are no guided modes in their infinite air cladding region. The distribution order of core modes does not change throughout the $r_{\mathrm{co}}$ range as: $\mathrm{LP}_{01}, \mathrm{LP}_{11}$, $\mathrm{LP}_{21}, \ldots$, with decreasing $n_{\text {eff }}$ as shown in the region between two red dash horizontal lines in Fig. 2(a).

In Ref. [1] the mode reorganization phenomenon of the $\mathrm{LP}_{0 \mathrm{n}}$ mode was discussed, where when one cladding-
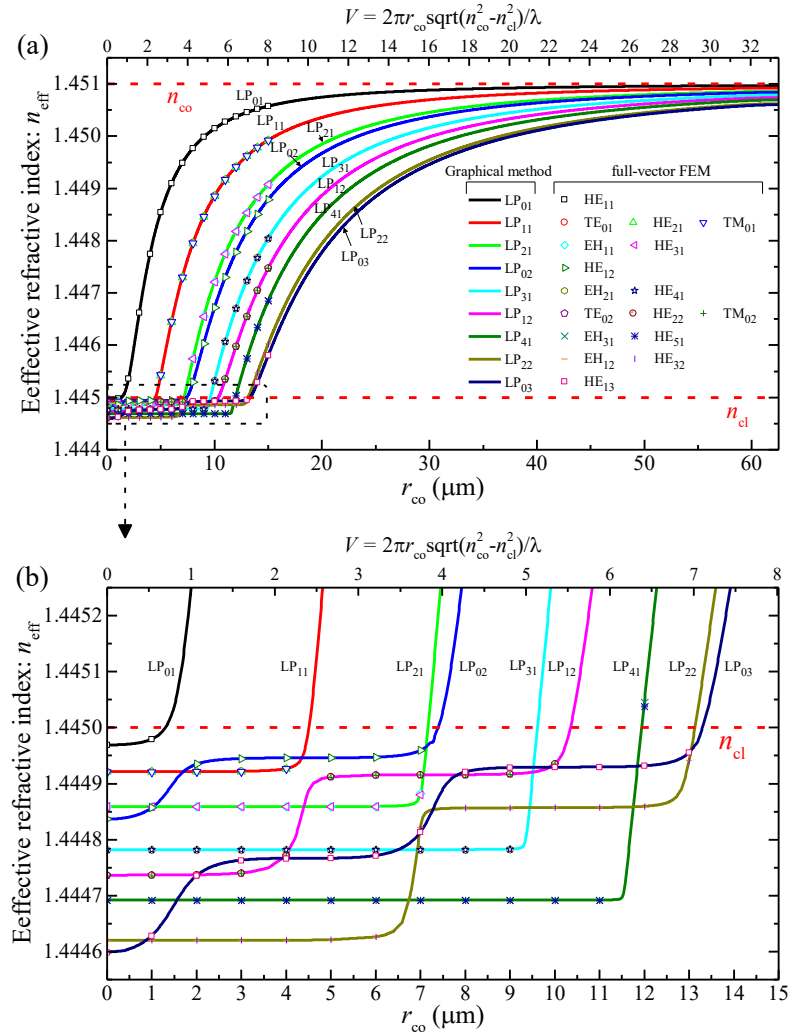

Fig. 2. (a) Dispersion curves $\left(n_{\text {eff }}\right.$ vs. $\left.r_{\text {co }}\right)$ of the modes in step-index optical fibers. (b) is a partially enlarged graph of (a), where the part is indicated by a black dash rectangle. The scalar $\mathrm{LP}_{\mathrm{mn}}$ modes indicated by the solid curves are calculated by graphical method. The vector modes $\left(\mathrm{TE}_{0 \mathrm{n}}, \mathrm{TM}_{0 \mathrm{n}}, \mathrm{HE}_{\mathrm{mn}}\right.$ and $\left.\mathrm{EH}_{\mathrm{mn}}\right)$ indicated by the scattered curves are calculated by the full-vector FEM.

type mode $\mathrm{LP}_{0 \mathrm{n}}$ becomes a core-type mode the remaining cladding modes $L_{0}$ are reorganized. The phenomenon also occurs for the remaining $\mathrm{LP}_{\mathrm{mn}}$ modes. The mode reorganization causes the step-like changes in the $n_{\text {eff }}$ values for the cladding modes, as shown in Fig. 2(b). Compared to the core modes, the distribution order of the cladding modes changes with the core radius $r_{\mathrm{co}}$. It should be noted that only the modes with the same azimuthal number $\mathrm{m}$ are reorganized as the mode transition of the lower order mode occurs. For example, when the cladding-type $\operatorname{LP}_{01}(\mathrm{~m}=0)$ becomes a core-type mode, all the $\mathrm{LP}_{0 \mathrm{n}}(\mathrm{m}=0, \mathrm{n}>1)$ modes are reorganized while the other $\mathrm{LP}_{\mathrm{mn}}(\mathrm{m} \neq 0)$ modes are not affected. In the whole range from $r_{\mathrm{co}}=0 \mu \mathrm{m}$ to the cutoff point as shown in the dispersion region below the red dash horizontal line at $n_{\mathrm{cl}}$, the number of times such reorganizations occur (the number of $n_{\text {eff }}$ step changes on the diagram Fig. 2(b)) for a single cladding mode $\mathrm{LP}_{\mathrm{mn}}$ is the same as the radial number $n$. For example, LP $_{01}$ experiences a single mode reorganization process, while the $\mathrm{LP}_{02}$ mode experiences the reorganization twice.

The cutoff for the $\mathrm{LP}_{01}\left(\mathrm{HE}_{11}\right)$ mode occurs circa a value for $r_{\mathrm{co}}=1.3 \mu \mathrm{m}(V=0.7214)$, as shown as the intersect point of the dispersion curve and the red dash horizonal line at $n_{\text {eff }}=n_{\mathrm{cl}}$. Clearly, there is no core-type mode when $r_{\mathrm{co}}<1.3 \mu \mathrm{m}(V<0.7214)$. The cutoff point for $\mathrm{LP}_{11}\left(\mathrm{TE}_{01}, \mathrm{TM}_{01}\right.$ and $\left.\mathrm{HE}_{21}\right)$ is at about $r_{\mathrm{co}}=4.5 \mu \mathrm{m}$ 


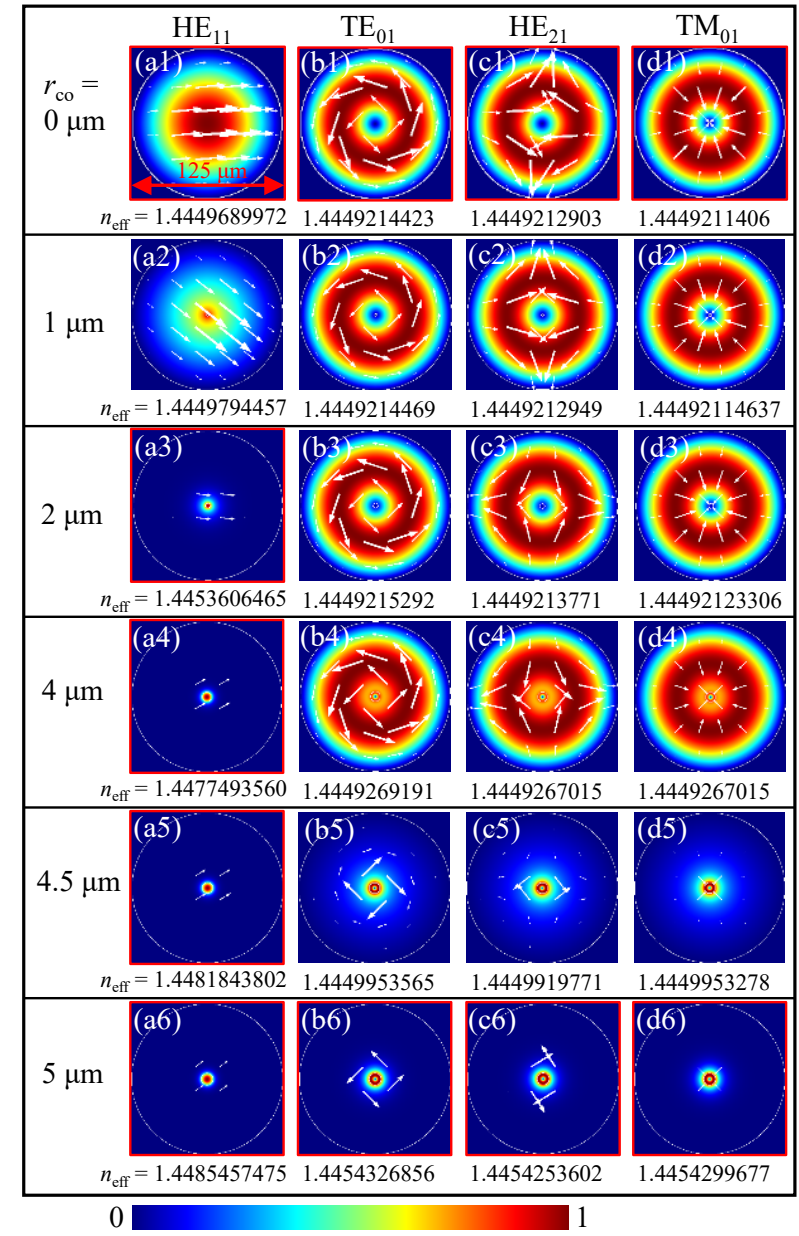

Fig. 3. Modal intensities and electric field vector distributions of the $\mathrm{HE}_{11}, \mathrm{TE}_{01}, \mathrm{HE}_{21}$ and $\mathrm{TM}_{01}$ modes, with several different $r_{\mathrm{co}}$ as indicated on the left. The modes in the no-core fibers $\left(r_{\mathrm{co}}=0 \mu \mathrm{m}\right)$, shown in a1, b1 c1 and d1, are core-type modes; the modes shown in a3-a6, b6, c6 and d6 are also core-type modes for $n_{\text {eff }}>1.445$; the rest are cladding-type modes with $n_{\text {eff }}<1.445$. (The core-type modes are indicated by the red frames)

$(V=2.40456)$, which is close to the value $(V=2.40483)$ calculated with a two-layer step-index fiber model.

The modal intensity distributions of $\mathrm{LP}_{\text {on }}$ modes were analyzed in our previous paper [1]. Fig. 3 shows the intensities and electric field vector distributions of the vector modes $\mathrm{HE}_{11}$ (corresponding to the scalar mode $\left.\mathrm{LP}_{01}\right), \mathrm{TE}_{01}, \mathrm{HE}_{21}$, and $\mathrm{TM}_{01}$ modes $\left(\mathrm{LP}_{11}\right)$ for several core radii, calculated by the full-vector FEM. The core-type mode field distributions are highlighted with red frames, while the remaining unhighlighted contour plots represent cladding-type modes. All the modes in an NCF $\left(r_{\mathrm{co}}=0 \mu \mathrm{m}\right)$ are core-type modes as shown in Figs. 3(a1), 3(b1), 3(c1) and 3(d1), where the intensity is extended throughout the whole fiber cross section. As the value of $r_{\mathrm{co}}$ increases from 0 to $1 \mu \mathrm{m}$, the $n_{\text {eff }}$ of mode $\mathrm{HE}_{11}$ increases while their central intensity area reduces as shown in Figs. 3(a1) and 3(a2). The mode $\mathrm{HE}_{11}$ becomes a core-type mode beyond the value of $r_{\mathrm{co}}$ $=1.3 \mu \mathrm{m}$. As shown in Figs. 3(a3)-3(a6), the energy of the $\mathrm{HE}_{11}$ mode is concentrated mainly inside the fiber core. The intensity distribution of $\mathrm{TE}_{01}, \mathrm{HE}_{21}$, and $\mathrm{TM}_{01}$ modes change very little for $r_{\mathrm{co}}<4 \mu \mathrm{m}$, corresponding to the almost horizontal dispersion line in Fig. 2(b). They become the core-type modes as the core radius $r_{\mathrm{co}}$ increases up to $4.5 \mu \mathrm{m}$. The modes $\mathrm{TE}_{01}, \mathrm{HE}_{21}$ and $\mathrm{TM}_{01}$ have similar intensity distributions and $n_{\text {eff, }}$ therefore, they can be grouped as a $\mathrm{LP}_{11}$ mode.

\section{CONCLUSION}

The effective refractive indices of both the core and the cladding modes in three-layer step-index optical fibers as a function of core radius were calculated independently by the graphical method and the full-vector FEM. Due to the fields being far from the cutoff approximation in the case of the no-core fiber and the small index difference between the core and cladding in the other cases, the vector modes $\left(\mathrm{TE}_{0 \mathrm{n}}, \mathrm{TM}_{0 \mathrm{n}}, \mathrm{HE}_{\mathrm{mn}}\right.$ and $\mathrm{EH}_{\mathrm{mn}}$ ) are perfect degeneracies and can be grouped into the corresponding $\mathrm{LP}_{\mathrm{mn}}$ modes. The transition from a cladding-type to a core-type mode has been studied. Compared to the unchanged distribution order of the core-type modes, the distribution order of cladding-type modes changes with the core radius $r_{\mathrm{co}}$. The cladding modes show mode reorganization characteristics, indicating a different dispersion behavior compared to that of the core modes. The distinct properties of cladding modes deserve further study in the field of optical fiber technologies.

\section{ACKNOWLEDGMENTS}

This work was supported by Technological University Dublin under the FIOSRAIGH 2016 scholarship program.

\section{REFERENCES}

[1] X. Lian, Q. Wu, G. Farrell, C. Shen, Y. Ma, and Y. Semenova, "Discrete self-imaging in small-core optical fiber interferometers", J. Lightw. Technol., vol. 37, no. 9, pp. 1873-1884, 2019.

[2] J. A. Buck, Fundamentals of Optical Fibers. Hoboken, NJ, USA: Wiley, 2004.

[3] C. Tsao, Optical Fibre Waveguide Analysis. London, U.K.: Oxford Univ. Press, 1992.

[4] M. Monerie, "Propagation in doubly clad single-mode fibers," IEEE Trans. Microw. Theory Techn., vol. MTT-30, no. 4, pp. 381-388, Apr. 1982.

[5] T. Erdogan, "Cladding-mode resonances in short-and long-period fiber grating filters", JOSA A, vol. 14, no. 8, pp. 1760-1773, 1997.

[6] O. V. Ivanov, S. A. Nikitov, and Y. V. Gulyaev, "Cladding modes of optical fibers: properties and applications," Phys. Uspekhi, vol. 49, no. 2, pp. 167-191, 2006.

[7] M. J. Digonnet, H. K. Kim, G. S. Kino, and S. Fan, "Understanding air-core photonic-bandgap fibers: Analogy to conventional fibers", J. Lightw. Technol., vol. 23, no. 12 , pp. 4169-4177, 2005. 